\title{
Impact of different methods on the detection frequency of BRAF mutation in Papillary Thyroid Carcinoma (PTC)
}

Artur Kowalik ${ }^{1}$, Aldona Kowalska ${ }^{2}$, Janusz Kopczyński ${ }^{3}$ Agnieszka Walczyk ${ }^{2}$ Ewelina Nowak ${ }^{1}$ Elżbieta Wypiórkiewicz ${ }^{1}$, Renata Chodurska ${ }^{1}$, Liliana Pięciak ${ }^{1}$, Stanisław Góźdź ${ }^{4-5}$

${ }^{1}$ Department of Molecular Diagnostics, ${ }^{2}$ Department of Endocrinology and Nuclear Medicine, ${ }^{3}$ Department of Surgical Pathology, ${ }^{4}$ Department of Chemotherapy, Holycross Cancer Centre, Kielce, Poland

${ }^{5}$ Faculty of Health Sciences, Jan Kochanowski University, Kielce, Poland

\section{INTRODUCTION}

Still there is a discussion about the importance of diagnostic and prognostic relevance of $\mathrm{V} 600 \mathrm{E}$ mutation in the BRAF gene. The wide range of $B R A F^{\mathrm{V} 600 \mathrm{E}}$ mutation frequency (40\% to $80 \%$ ) does not facilitate this task. Population diversity and genotyping methodology are suspected sources of differences seen in the mutation frequencies detected by different studies. The aim of this study was to assess the prevalence of mutations in the BRAF ${ }^{\mathrm{V} 600 \mathrm{E}}$, depending on the diagnostic method used (direct sequencing and ASA -PCR) analyzed on a Holycross population .

\section{MATERIALS AND METHODS}

Tumors from 411 consecutive patients with PTC genotyped for BRAF ${ }^{\mathrm{V} 600 E}$ using capillary sequencing and allele specific amplification PCR (ASA-PCR). DNA subjected to genotyping isolated following macrodissection using tissue area marked by the pathologist. The level of sensitivity of the capillary sequencing is generally estimated at 10$20 \%$ of mutated allele in WT alleles and the sensitivity of the ASA-PCR technique is about $1 \%$ of mutated allele in WT alleles.

\section{RESULTS}

Of the 411 cases genotyped using both methods capillary sequencing and ASA-PCR BRAF ${ }^{\mathrm{V} 600 \mathrm{E}}$ mutation detected in 270 cases $(65.7 \%)$ Fig. $1 \mathrm{~A}, 2 \mathrm{i} 3 \mathrm{~A}$. Additionally detected $8(1.9 \%)$ other mutations in the BRAF near codon V600 Fig. 3A . In total, this gives $278(67.6 \%)$ cases with mutation Fig. 3A. In contrast, no mutations were found in 121 $(29.4 \%)$ cases Fig. 1B i 3A. Because of DNA degradation we failed to get a result in $12(2.9 \%)$ cases Fig. 3A. If we take into account only the results obtained by sequencing, $\mathrm{BRAF}^{\mathrm{V} 600 \mathrm{E}}$ mutation detected in $186(45.3 \%)$ cases and $8(1.9 \%)$ mutations in the vicinity of codon BRAF ${ }^{V 600 E}$ Fig. 3B. In total sequencing detected $194(47.2 \%)$ mutations in the BRAF Fig. 3B . In $151(36.7 \%)$ cases no mutation detected Fig 1B i 3B. In $31(7.5 \%)$ cases sequencing result was inconclusive Fig. 1C i 3B . DNA degradated in 35 $(8.5 \%)$ cases and we didn't obtained the result Fig. 3B. However, with the ASA -PCR method BRAF ${ }^{\mathrm{V} 600 \mathrm{E}}$ mutations were found in 270 (65.7\%) cases Fig. 2. This method only detects the V600E mutation, so it hasn't detected eight cases with mutations located in the vicinity of the BRAF V600 codon . In 27 (6,6\%) cases the DNA was degraded , and one result was inconclusive Fig. $3 \mathrm{C}$

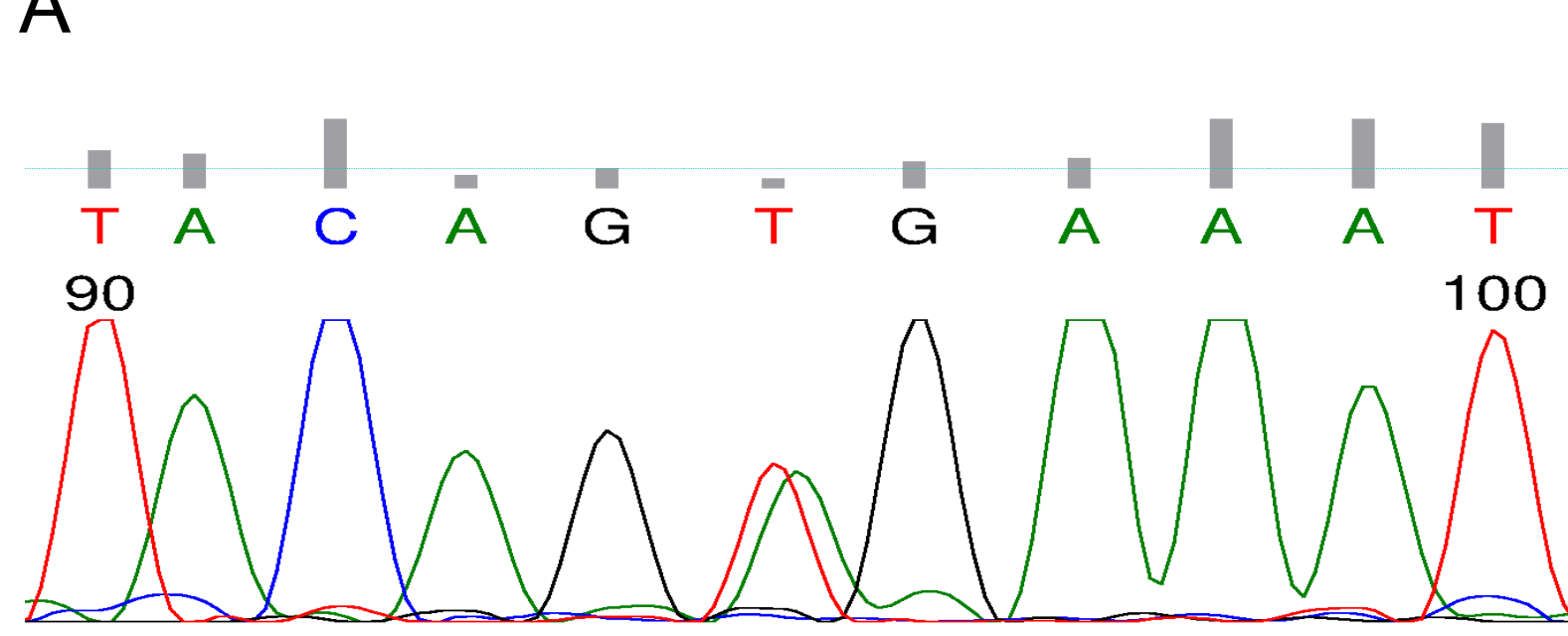

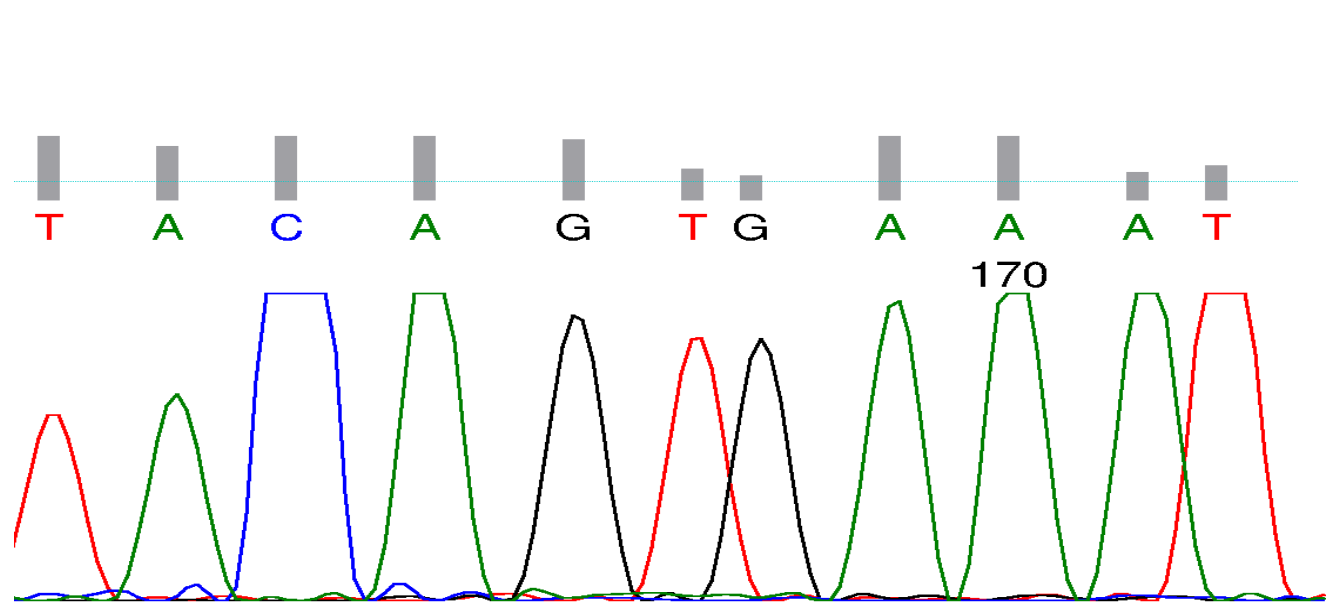

C

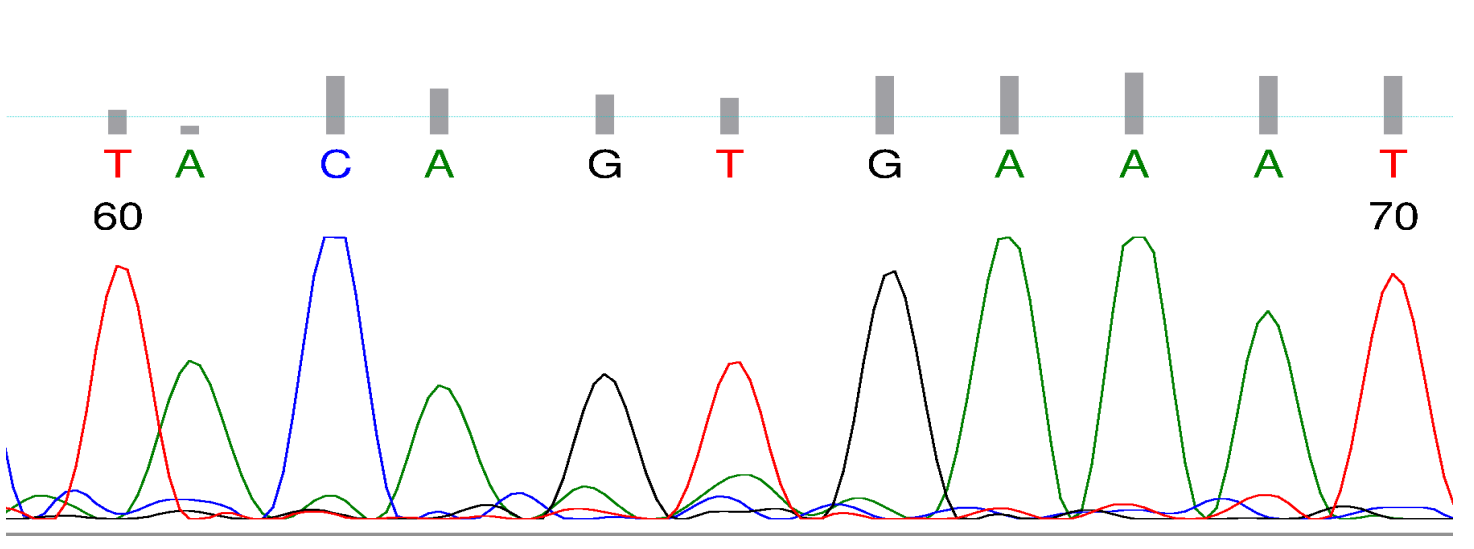

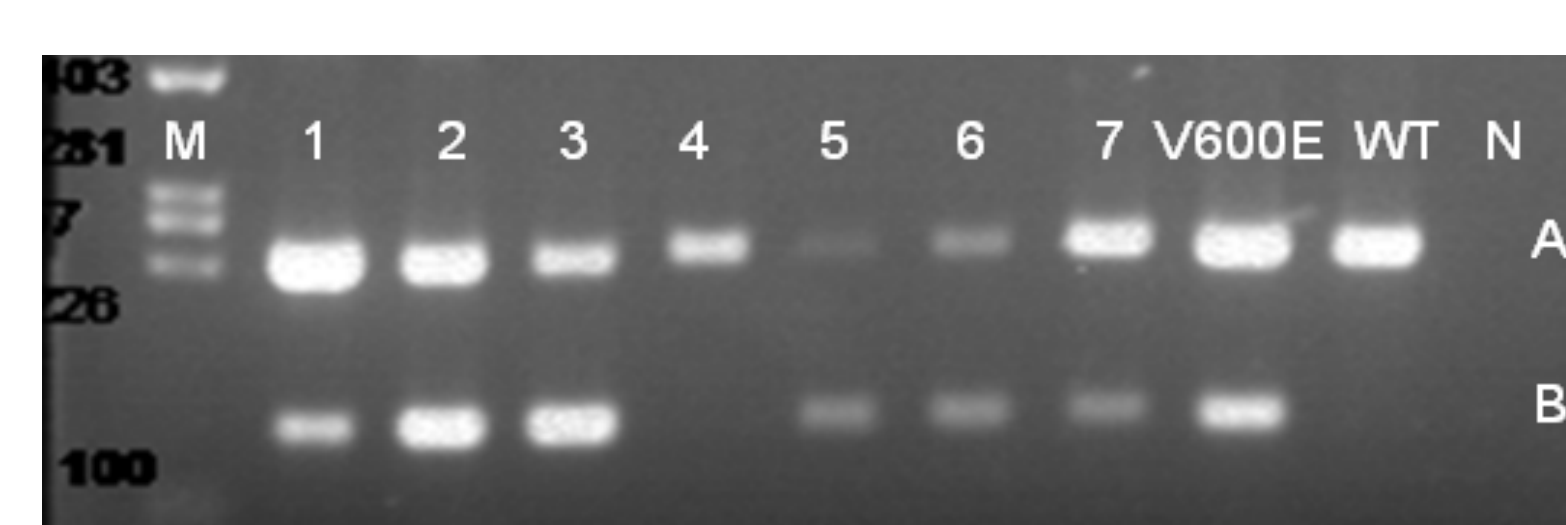

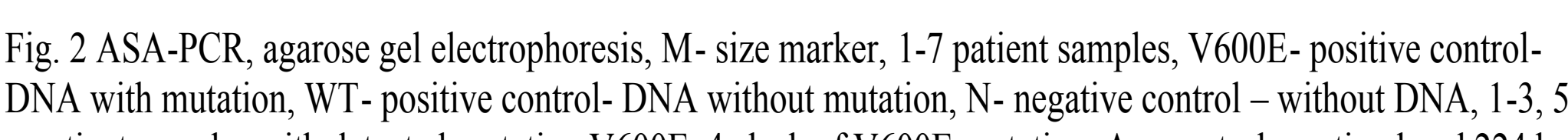
- patient samples with detected mutation $V 600 \mathrm{D}$, 4 - lack of $\mathrm{V}$.
B- band informing about presence of mutation $\mathrm{V} 600 \mathrm{E}$
112 bp.
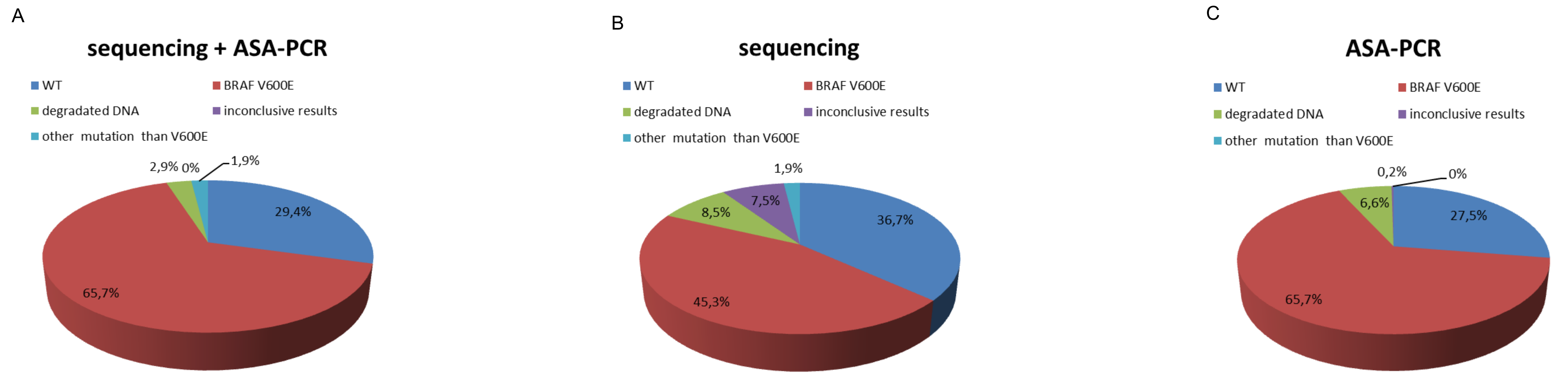

Fig. 3 Pie charts shows distribution of genotypng results according to methods used

\section{CONCLUSIONS}

The frequency of detected mutations in the BRAF depends on the method. The combination of methods with different sensitivity and spectrum of detected mutations increases the incidence of PTC with the presence of mutations in BRAF. Differences in the frequency of BRAF mutations in PTC described the literature may be the result of applied research methodology. This might be attributed to the tumor clonallity phenomenon. It is recommended to use a combination of methods in the diagnosis of mutations in the BRAF PTC. 